\title{
Synchronization of Intermittently Coupled Dynamical Networks
}

\author{
Gang Zhang' and Guanrong Chen ${ }^{2}$ \\ ${ }^{1}$ College of Mathematics and Information Science, Hebei Normal University, Shijiazhuang 050016, China \\ ${ }^{2}$ Department of Electronic Engineering, City University of Hong Kong, Kowloon, Hong Kong \\ Correspondence should be addressed to Gang Zhang; gzhang_math@163.com
}

Received 17 April 2013; Accepted 5 June 2013

Academic Editor: Wenwu Yu

Copyright (C) 2013 G. Zhang and G. Chen. This is an open access article distributed under the Creative Commons Attribution License, which permits unrestricted use, distribution, and reproduction in any medium, provided the original work is properly cited.

\begin{abstract}
This paper investigates the synchronization phenomenon of an intermittently coupled dynamical network in which the coupling among nodes can occur only at discrete instants and the coupling configuration of the network is time varying. A model of intermittently coupled dynamical network consisting of identical nodes is introduced. Based on the stability theory for impulsive differential equations, some synchronization criteria for intermittently coupled dynamical networks are derived. The network synchronizability is shown to be related to the second largest and the smallest eigenvalues of the coupling matrix, the coupling strength, and the impulsive intervals. Using the chaotic Chua system and Lorenz system as nodes of a dynamical network for simulation, respectively, the theoretical results are verified and illustrated.
\end{abstract}

\section{Introduction}

A complex dynamical network is a large set of interconnected nodes, in which each node is typically a nonlinear dynamical system. Many real systems in nature and engineering, such as physical, biological, technological, and social systems, can be described by various models of complex dynamical networks. Complex dynamical networks, therefore, have become a significant research topic for studying nonlinear dynamics in various fields of sciences and humanities today [1-7].

One of the most remarkable phenomena in complex dynamical networks is the synchronization of dynamical nodes, which has been extensively investigated in recent years [8-32]. Wang and Chen $[8,9]$ presented a unified dynamical network model and investigated its synchronization in smallworld and scale-free networks. Belykh et al. [11, 12] proposed an effective method for determining the global stability of synchronization in dynamical networks with different topologies, which combines the Lyapunov function approach with graph-theoretic reasoning. Restrepo et al. [19] studied the emergence of coherence in large-scale complex networks of interacting heterogeneous dynamical systems and showed that the largest eigenvalue of the network adjacency matrix plays a key role in determining the transition to coherence.
Zhou et al. [20-22] derived some synchronization criteria for general complex delayed dynamical networks. Recently, synchronization of complex dynamical networks with impulsive control has been extensively studied [23-32]. For example, Guan et al. $[23,24]$ proposed a hybrid impulsive and switching control strategy and investigated the stabilization of complex networks. Liu et al. [25] proposed an impulsive synchronization scheme for an uncertain dynamical network. Zhang et al. [26] designed an effective impulsive controller to achieve impulsive synchronization for a complex dynamical network with unknown coupling. Lu et al. [27] investigated the problem of globally exponential synchronization of impulsive dynamical network, with a unified impulsive synchronization criterion derived by proposing a concept of average impulsive interval. Tang et al. [29] investigated the pinning synchronization problem of stochastic impulsive networks. Zhou et al. [30] proposed an impulsive control approach for analyzing pinning stability in a complex delayed dynamical network comprised of linearly coupled dynamical systems with coupling delays. Han et al. [31] and Sun et al. [32] derived some distributed impulsive control schemes for various impulsively coupled complex dynamical systems with or without delays. 
However, the aforementioned work and most other existing research focus on the networks whose couplings are invariable or continuously varying in time. In the real world, there are many networks in which the coupling among nodes is intermittent. For example, in neural networks, the connections among neurons are usually cut-off type or extremely faint. There are prominent impulsive interactions among neurons when they are stimulated by certain signals, and the interactions are not identical due to the differences of stimulating signals. This type of networks is referred to as intermittently coupled dynamical networks. Note that the structure of such networks is time varying, and the interactions among nodes can only take place when some conditions are satisfied which are usually described by a group of discrete time sequences. To the best of our knowledge, there are few theoretical results about intermittently coupled dynamical networks in the current literature.

In this paper, an intermittently coupled dynamical network consisting of identical nodes is investigated. In the network, the coupling among nodes can only occur at discrete instants, and the coupling configuration of the network is varying at different instants. Based on the stability theory for impulsive differential equations, some synchronization criteria are obtained, showing that the synchronizability of the intermittently coupled dynamical network is related to the second largest and the smallest eigenvalues of the coupling matrix, the coupling strength, and the impulsive intervals. It turns out that the analytical results about the second largest and the smallest eigenvalues of the coupling matrix are consistent with other known results about complex dynamical networks $[8,9,19]$.

The rest of the paper is organized as follows. In Section 2, an intermittently coupled dynamical network model is introduced, and some necessary definitions and preliminary lemmas are presented. The main results of the paper are given in Section 3, where some synchronization criteria for the network model are derived. In Section 4, using the chaotic Chua system and Lorenz system as nodes of a dynamical network, respectively, numerical simulations are performed to illustrate and verify the theoretical results. Finally, conclusions are drawn in Section 5.

\section{Model Description and Preliminaries}

Consider an intermittently coupled dynamical network consisting of $N$ identical nodes, with each node being an $n$ dimension dynamical system, described by

$$
\begin{array}{r}
\dot{x}_{i}(t)=f\left(x_{i}(t)\right)+\sum_{k=1}^{\infty} U_{i}\left(t ; x_{1}, \ldots, x_{N}\right) \delta\left(t-t_{k}\right), \\
i=1,2, \ldots, N,
\end{array}
$$

where $x_{i}=\left(x_{i 1}, x_{i 2}, \ldots, x_{i n}\right)^{T} \in R^{n}$ is the state vector of node $i, f: R^{n} \rightarrow R^{n}$ is continuously differentiable, $U_{i}\left(t ; x_{1}, x_{2}, \ldots, x_{N}\right): R \times R^{n N} \rightarrow R^{n}$ are coupling functions, time sequence $\left\{t_{k}\right\}_{k=1}^{\infty}$ satisfies $0<t_{1}<t_{2}<\cdots<t_{k}<t_{k+1}$ $<\cdots, \lim _{k \rightarrow \infty} t_{k}=\infty$, and $\delta(\cdot)$ is a Dirac delta function defined by

$$
\delta\left(t-t_{k}\right)= \begin{cases}\infty, & t=t_{k} \\ 0, & t \neq t_{k}\end{cases}
$$

satisfying the identities $\int_{-\infty}^{+\infty} \delta(t) d t=1$ and $\int_{-\infty}^{+\infty} g(t) \delta(t-$ $\left.t_{0}\right) d t=g\left(t_{0}\right)$ for any continuous function $g(t)$. Suppose that the function $f$ satisfies the following condition.

(A1) There exists a positive scalar $L$ such that, for any $x, y \in R^{n}$,

$$
\|f(x)-f(y)\| \leq L\|x-y\|
$$

In this paper, we consider the dynamical network with timevarying diffusive coupling at discrete instants. Let

$$
U_{i}\left(t_{k} ; x_{1}, \ldots, x_{N}\right)=c \sum_{j=1, j \neq i}^{N} a_{i j}\left(t_{k}\right) \Gamma\left[x_{j}\left(t_{k}\right)-x_{i}\left(t_{k}\right)\right],
$$

where constant $c>0$ is the coupling strength, $A\left(t_{k}\right)=$ $\left(a_{i j}\left(t_{k}\right)\right) \in R^{N \times N}$ is the outer-coupling matrix which represents the coupling configuration of network (1) at instant $t_{k}$, and $a_{i j}\left(t_{k}\right)$ is defined as follows: if there is a connection between nodes $i$ and $j(i \neq j)$, then $a_{i j}\left(t_{k}\right)=a_{j i}\left(t_{k}\right)=1$, otherwise, $a_{i j}\left(t_{k}\right)=a_{j i}\left(t_{k}\right)=0(i \neq j)$; the diagonal elements are defined by

$$
\begin{array}{r}
a_{i i}\left(t_{k}\right)=-\sum_{j=1, j \neq i}^{N} a_{i j}\left(t_{k}\right)=-\sum_{j=1, j \neq i}^{N} a_{j i}\left(t_{k}\right), \\
i=1,2, \ldots, N,
\end{array}
$$

and $\Gamma=\operatorname{diag}\left\{\gamma_{1}, \gamma_{2}, \ldots, \gamma_{n}\right\} \in R^{n \times n}$ is the inner-linking matrix. Let $\Gamma=\operatorname{diag}\{1,1, \ldots, 1\}$. Thus, (4) becomes

$$
U_{i}\left(t_{k} ; x_{1}, x_{2}, \ldots, x_{N}\right)=c \sum_{j=1}^{N} a_{i j}\left(t_{k}\right) \Gamma x_{j}\left(t_{k}\right)
$$

Assume that network (1) is connected at instants $t_{1}, t_{2}, \ldots$, in the sense that there is no isolated cluster; that is, $A\left(t_{k}\right)$ is an irreducible matrix.

Lemma 1 (see [14]). Suppose that the outer-coupling matrix $A=\left(a_{i j}\right) \in R^{N \times N}$ satisfies the above-mentioned conditions. Then,

(i) 0 is an eigenvalue of matrix A of multiplicity 1 , associated with eigenvector $(1 / \sqrt{N}, 1 / \sqrt{N}, \ldots, 1 / \sqrt{N})^{T}$;

(ii) all the other eigenvalues of $A$ are real-valued and are strictly negative.

Lemma 1 implies that the outer-coupling matrix $A\left(t_{k}\right)$ of network (1) at instant $t_{k}$ has a 0 eigenvalue of multiplicity 1 , with $\lambda_{N}\left(t_{k}\right) \leq \cdots \leq \lambda_{2}\left(t_{k}\right)<\lambda_{1}\left(t_{k}\right)=0$. 
Remark 2. According to matrix theory, there exists an orthogonal matrix $B\left(t_{k}\right)$ such that $A\left(t_{k}\right)=B^{T}\left(t_{k}\right) \Lambda\left(t_{k}\right) B\left(t_{k}\right)$, where $\Lambda\left(t_{k}\right)=\operatorname{diag}\left\{\lambda_{1}\left(t_{k}\right), \ldots, \lambda_{N}\left(t_{k}\right)\right\}$. It is easy to see that the first column of $B^{T}\left(t_{k}\right)$ can be chosen as the eigenvector $(1 / \sqrt{N}, 1 / \sqrt{N}, \ldots, 1 / \sqrt{N})^{T}$ corresponding to the zero eigenvalue of $A\left(t_{k}\right)$.

Next, network (1) is rewritten as the following impulsive differential equations:

$$
\begin{gathered}
\dot{x}_{i}(t)=f\left(x_{i}(t)\right), \quad t \neq t_{k}, \quad i=1,2, \ldots, N, \\
\Delta x_{i}\left(t_{k}\right)=c \sum_{j=1}^{N} a_{i j}\left(t_{k}\right) x_{j}\left(t_{k}\right), \quad t=t_{k}, k=1,2, \ldots,
\end{gathered}
$$

where $\Delta x_{i}\left(t_{k}\right)=x_{i}\left(t_{k}^{+}\right)-x_{i}\left(t_{k}^{-}\right)$is the "jump" in the state variable at instant $t_{k}$, with $x_{i}\left(t_{k}^{+}\right)=\lim _{t \rightarrow t_{k}^{+}} x(t), x_{i}\left(t_{k}^{-}\right)=$ $\lim _{t \rightarrow t_{k}^{-}} x(t)$. For simplicity, assume that $x_{i}\left(t_{k}^{-}\right)=x_{i}\left(t_{k}\right)$, which means $x_{i}(t)$ is continuous from the left.

Definition 3. The synchronization manifold is presented as $\mathbf{S}=\left\{\left(x_{1}^{T}, x_{2}^{T}, \ldots, x_{N}^{T}\right)^{T} \in R^{n N}: x_{i}=x_{j}, i, j=1,2, \ldots N\right\}$, where $x_{i} \in R^{n}, i=1,2, \ldots, N$.

Definition 4. The coupled system (6) is said to achieve synchronization if, for all $i, j=1,2, \ldots, N, \lim _{t \rightarrow \infty} \| x_{i}(t)-$ $x_{j}(t) \|=0$.

From Definitions 3 and 4, it can be easily seen that the intermittently coupled system (7) achieves synchronization if and only if the synchronization manifold $\mathbf{S}$ for the coupled system (7) is globally asymptotically stable.

\section{Synchronization of the Intermittently Coupled Network}

Let $\bar{x}(t)=(1 / N) \sum_{j=1}^{N} x_{j}(t)$ and $\bar{X}(t)=\left[\bar{x}^{T}(t), \ldots, \bar{x}^{T}(t)\right]^{T} \epsilon$ $\mathbf{S}$, which can be regarded as a projection of $X(t)=$ $\left[x_{1}^{T}(t), \ldots, x_{N}^{T}(t)\right]^{T}$ on the synchronization manifold. The dynamical equation of $\bar{x}(t)$ can be written as

$$
\dot{\bar{x}}(t)=\frac{1}{N} \sum_{i=1}^{N} \dot{x}_{i}(t)=\frac{1}{N} \sum_{i=1}^{N} f\left(x_{i}(t)\right), \quad t \neq t_{k} .
$$

On the other hand,

$$
\begin{aligned}
\bar{x}\left(t_{k}^{+}\right) & =\frac{1}{N} \sum_{i=1}^{N} x_{i}\left(t_{k}^{+}\right) \\
& =\frac{1}{N} \sum_{i=1}^{N}\left[x_{i}\left(t_{k}\right)+c \sum_{j=1}^{N} a_{i j}\left(t_{k}\right) x_{j}\left(t_{k}\right)\right] \\
& =\frac{1}{N} \sum_{i=1}^{N} x_{i}\left(t_{k}\right)+\frac{c}{N} \sum_{j=1}^{N}\left[\sum_{i=1}^{N} a_{i j}\left(t_{k}\right)\right] x_{j}\left(t_{k}\right) \\
& =\bar{x}\left(t_{k}\right), \quad k=1,2, \ldots
\end{aligned}
$$

Define the synchronization error of node $i$ as $e_{i}(t)=x_{i}(t)-$ $\bar{x}(t), i=1,2, \ldots, N$. Obviously, $\sum_{i=1}^{N} e_{i}(t)=0$.
For $t \neq t_{k}, k=1,2, \ldots$, one has

$$
\begin{aligned}
\dot{e}_{i}(t) & =f\left(x_{i}(t)\right)-\frac{1}{N} \sum_{j=1}^{N} f\left(x_{i}(t)\right) \\
& =f\left(x_{i}(t)\right)-f(\bar{x}(t))+\frac{1}{N} \sum_{j=1}^{N}\left[f(\bar{x}(t))-f\left(x_{j}(t)\right)\right] \\
& =\tilde{f}\left(e_{i}(t)\right)-\frac{1}{N} \sum_{j=1}^{N} \tilde{f}\left(e_{j}(t)\right),
\end{aligned}
$$

where $\tilde{f}\left(e_{i}(t)\right)=f\left(\bar{x}(t)+e_{i}(t)\right)-f(\bar{x}(t))$.

According to assumption (A1), it can be easily shown that

$$
\left\|\tilde{f}\left(e_{i}(t)\right)\right\| \leq L\left\|e_{i}(t)\right\|, \quad i=1,2, \ldots, N .
$$

For $t=t_{k}, k=1,2, \ldots$, one has

$$
\begin{aligned}
e_{i}\left(t_{k}^{+}\right) & =x_{i}\left(t_{k}^{+}\right)-\bar{x}\left(t_{k}^{+}\right) \\
& =x_{i}\left(t_{k}\right)+c \sum_{j=1}^{N} a_{i j}\left(t_{k}\right) x_{j}\left(t_{k}\right)-\bar{x}\left(t_{k}\right) \\
& =e_{i}\left(t_{k}\right)+c \sum_{j=1}^{N} a_{i j}\left(t_{k}\right) e_{j}\left(t_{k}\right)
\end{aligned}
$$

Therefore, the error dynamical system can be described as

$$
\begin{aligned}
& \dot{e}_{i}(t)=\widetilde{f}\left(e_{i}(t)\right)-\frac{1}{N} \sum_{j=1}^{N} \widetilde{f}\left(e_{j}(t)\right), \quad t \neq t_{k}, \\
& e_{i}\left(t_{k}^{+}\right)=e_{i}\left(t_{k}\right)+c \sum_{j=1}^{N} a_{i j}\left(t_{k}\right) e_{j}\left(t_{k}\right), \quad k=1,2, \ldots,
\end{aligned}
$$

where $i=1,2, \ldots, N$.

Let $e(t)=\left[e_{1}^{T}(t), \ldots, e_{N}^{T}(t)\right]^{T} \in R^{n N}$. It is easily seen that the stability of the synchronization manifold is equivalent to $e(t) \rightarrow 0$ as $t \rightarrow \infty$. In the following, we directly investigate the dynamical behaviors of the error dynamical system (13).

Theorem 5. Let $P \in R^{n \times n}$ be a symmetric and positive definite matrix, with $\lambda_{\max }(P)$ and $\lambda_{\min }(P)$ being the largest and the smallest eigenvalues, respectively. Suppose that there exists a constant $\xi>1$ and, for all $k=1,2, \ldots$,

$$
\frac{\lambda_{\text {min }}(P) \exp ^{-2 L \sqrt{\left(\lambda_{\text {max }}(P) / \lambda_{\min }(P)\right)}\left(t_{k+1}-t_{k}\right)}}{\lambda_{\text {max }}(P) \lambda\left(t_{k}\right)} \geq \xi>1,
$$

where $\lambda\left(t_{k}\right)=\max \left\{\left(1+c \lambda_{i}\left(t_{k}\right)\right)^{2} \mid i=2, N\right\}, \lambda_{2}\left(t_{k}\right)$ and $\lambda_{N}\left(t_{k}\right)$ are the second largest and the smallest eigenvalues of matrix $A\left(t_{k}\right)$, respectively, and the constant $c>0$ is the coupling strength. Then, the trivial solution of the error dynamical system (21) is asymptotically stable, implying that network (1) achieves synchronization. 
Proof. Construct a Lyapunov function as

$$
V(t, e)=e^{T}(t)\left(I_{N} \otimes P\right) e(t)=\sum_{i=1}^{N} e_{i}^{T}(t) P e_{i}(t),
$$

where $\otimes$ denotes the Kronecker product operator.

For $t \neq t_{k}$, taking Dini's derivative along the trajectories of (13) gives

$$
\begin{aligned}
D^{+} V(t, e)= & \sum_{i=1}^{N} 2 e_{i}^{T}(t) P \dot{e}_{i}(t) \\
= & \sum_{i=1}^{N} 2 e_{i}^{T}(t) P\left[\tilde{f}\left(e_{i}(t)\right)-\frac{1}{N} \sum_{j=1}^{N} \tilde{f}\left(e_{j}(t)\right)\right] \\
= & \sum_{i=1}^{N} 2 e_{i}^{T}(t) P \tilde{f}\left(e_{i}(t)\right) \\
\leq & \sum_{i=1}^{N} \frac{1}{L} \sqrt{\lambda_{\min }(P) \lambda_{\max }(P)} \tilde{f}^{T}\left(e_{i}(t)\right) \tilde{f}\left(e_{i}(t)\right) \\
& +\frac{L}{\sqrt{\lambda_{\min }(P) \lambda_{\max }(P)}} e_{i}^{T}(t) P^{T} P e_{i}(t) \\
\leq & \sum_{i=1}^{N} L \sqrt{\frac{\lambda_{\min }(P) \lambda_{\max }(P)}{e_{i}^{T}(t) P e_{i}(t)}} \\
& +L \sqrt{\frac{\lambda_{\max }(P)}{\lambda_{\min }(P)}} e_{i}^{T}(t) P e_{i}(t) \\
= & 2 L \sqrt{\frac{\lambda_{\max }(P)}{\lambda_{\min }(P)}} V(t, e) .
\end{aligned}
$$

For $t=t_{k}$, one has

$$
\begin{aligned}
e\left(t_{k}^{+}\right) & =\left(e_{1}^{T}\left(t_{k}^{+}\right), e_{2}^{T}\left(t_{k}^{+}\right), \ldots, e_{N}^{T}\left(t_{k}^{+}\right)\right)^{T} \\
& =e\left(t_{k}\right)+c A^{T}\left(t_{k}\right) \otimes I_{n} e\left(t_{k}\right) \\
& =\left[I_{N} \otimes I_{n}+c A^{T}\left(t_{k}\right) \otimes I_{n}\right] e\left(t_{k}\right) .
\end{aligned}
$$

Therefore,

$$
\begin{aligned}
V\left(t_{k}^{+}, e\right)= & e^{T}\left(t_{k}^{+}\right)\left(I_{N} \otimes P\right) e\left(t_{k}^{+}\right) \\
= & e^{T}\left(t_{k}\right)\left[\left(I_{N}+c A\left(t_{k}\right)\right) \otimes I_{n}\right]^{T}\left(I_{N} \otimes P\right) \\
& \times\left[\left(I_{N}+c A\left(t_{k}\right)\right) \otimes I_{n}\right] e\left(t_{k}\right) \\
\leq & \lambda_{\max }(P) e^{T}\left(t_{k}\right)\left[\left(I_{N}+c A\left(t_{k}\right)\right) \otimes I_{n}\right]^{T} \\
& \times\left[\left(I_{N}+c A\left(t_{k}\right)\right) \otimes I_{n}\right] e\left(t_{k}\right) \\
= & \lambda_{\max }(P) e^{T}\left(t_{k}\right)\left[\left(I_{N}+c A\left(t_{k}\right)\right)^{2} \otimes I_{n}\right] e\left(t_{k}\right) .
\end{aligned}
$$

Based on the property of the matrix $A\left(t_{k}\right)$ mentioned in Remark 2, there exits an orthogonal matrix $B\left(t_{k}\right)$ such that $A\left(t_{k}\right)=B^{T}\left(t_{k}\right) \Lambda\left(t_{k}\right) B\left(t_{k}\right)$, where

$$
\Lambda\left(t_{k}\right)=\operatorname{diag}\left\{\lambda_{1}\left(t_{k}\right), \ldots, \lambda_{N}\left(t_{k}\right)\right\}
$$

and the first column of $B^{T}\left(t_{k}\right)$ is the eigenvector $(1 / \sqrt{N}$, $\ldots, 1 / \sqrt{N})^{T}$ corresponding to the zero eigenvalue of $A\left(t_{k}\right)$. Let $\eta\left(t_{k}\right)=\left(\eta_{1}^{T}\left(t_{k}\right), \ldots, \eta_{N}^{T}\left(t_{k}\right)\right)^{T}=\left(B\left(t_{k}\right) \otimes I_{n}\right) e\left(t_{k}\right)$. Then,

$$
\eta_{1}\left(t_{k}\right)=\frac{1}{\sqrt{N}} \sum_{j=1}^{N} e_{j}\left(t_{k}\right)=\mathbf{0} .
$$

Therefore,

$$
\eta\left(t_{k}\right)=\left(\mathbf{0}, \eta_{2}^{T}\left(t_{k}\right), \ldots, \eta_{N}^{T}\left(t_{k}\right)\right)^{T} .
$$

Let $\lambda\left(t_{k}\right)=\max \left\{\left(1+c \lambda_{i}\left(t_{k}\right)\right)^{2} \mid i=2, N\right\}$, where $\lambda_{2}\left(t_{k}\right)$ and $\lambda_{N}\left(t_{k}\right)$ are the second largest and the smallest eigenvalues of matrix $A\left(t_{k}\right)$, respectively. It follows from (18) and (21) that

$$
\begin{aligned}
V\left(t_{k}^{+}, e\right) \leq & \lambda_{\max }(P) e^{T}\left(t_{k}\right) \\
& \times\left[\left(B^{T}\left(t_{k}\right)\left(I_{N}+c \Lambda\left(t_{k}\right)\right)^{2} B\left(t_{k}\right)\right) \otimes I_{n}\right] e\left(t_{k}\right) \\
= & \lambda_{\max }(P) \eta^{T}\left(t_{k}\right)\left(\left(I_{N}+c \Lambda\left(t_{k}\right)\right)^{2} \otimes I_{n}\right) \eta\left(t_{k}\right) \\
= & \lambda_{\max }(P) \sum_{i=1}^{N} \eta_{i}^{T}\left(t_{k}\right)\left(1+c \lambda_{i}\left(t_{k}\right)\right)^{2} \eta_{i}\left(t_{k}\right) \\
\leq & \lambda_{\max }(P) \lambda\left(t_{k}\right) \sum_{i=1}^{N} \eta_{i}^{T}\left(t_{k}\right) \eta_{i}\left(t_{k}\right) \\
= & \lambda_{\max }(P) \lambda\left(t_{k}\right) e^{T}\left(t_{k}\right)\left(B^{T}\left(t_{k}\right) \otimes I_{n}\right) \\
& \times\left(B\left(t_{k}\right) \otimes I_{n}\right) e\left(t_{k}\right) \\
\leq & \frac{\lambda_{\max }(P)}{\lambda_{\min }(P)} \lambda\left(t_{k}\right) e^{T}\left(t_{k}\right)\left(I_{N} \otimes P\right) e\left(t_{k}\right) \\
= & \frac{\lambda_{\max }(P)}{\lambda_{\min }(P)} \lambda\left(t_{k}\right) V\left(t_{k}, e\right) .
\end{aligned}
$$

Let $S_{\rho}=\left\{x \in R^{n}:\|x\|<\rho\right\}$. Since $\left\|e\left(t_{k}^{+}\right)\right\|=\|\left[\left(I_{N}+c A\left(t_{k}\right)\right) \otimes\right.$ $\left.I_{n}\right] e\left(t_{k}\right)\|\leq\|\left(I_{N}+c A\left(t_{k}\right)\right) \otimes I_{n}\|\| e\left(t_{k}\right) \|$ and $\|\left(I_{N}+c A\left(t_{k}\right)\right) \otimes$ $I_{n} \|<\infty$, there exists a $\rho_{0}>0$ such that $e\left(t_{k}\right) \in S_{\rho_{0}}$ implies $e\left(t_{k}^{+}\right) \in S_{\rho}$ for all $k$.

Since $P$ is a symmetric and positive definite matrix, one has

$$
\lambda_{\min }(P) \sum_{i=1}^{n} e_{i}^{T}(t) e_{i}(t) \leq V(t, e) \leq \lambda_{\max }(P) \sum_{i=1}^{n} e_{i}^{T}(t) e_{i}(t) .
$$

Thus, by the well-known comparison Theorem [33], the asymptotic stability of the trivial solution of the impulsive 
dynamical system (13) follows from the comparison system below:

$$
\begin{aligned}
& \dot{\omega}(t)=2 L \sqrt{\frac{\lambda_{\text {max }}(P)}{\lambda_{\text {min }}(P)}} \omega(t), \quad t \neq t_{k}, \\
& \omega\left(t_{k}^{+}\right)=\frac{\lambda_{\max }(P)}{\lambda_{\min }(P)} \lambda\left(t_{k}\right) \omega\left(t_{k}\right), \quad t=t_{k}, k=1,2, \ldots, \\
& \omega\left(t_{0}^{+}\right)=\omega_{0} .
\end{aligned}
$$

Therefore, by the stability criterion for impulsive differential equations [34], there exists a constant $\xi>1$ such that

$$
\ln \frac{\lambda_{\text {max }}(P)}{\lambda_{\min }(P)} \lambda\left(t_{k}\right) \xi+2 L \sqrt{\frac{\lambda_{\text {max }}(P)}{\lambda_{\text {min }}(P)}}\left(t_{k+1}-t_{k}\right) \leq 0,
$$

namely,

$$
\frac{\lambda_{\min }(P) \exp ^{-2 L \sqrt{\left(\lambda_{\max }(P) / \lambda_{\min }(P)\right)}\left(t_{k+1}-t_{k}\right)}}{\lambda_{\max }(P) \lambda\left(t_{k}\right)} \geq \xi>1 .
$$

Hence, the trivial solution of error dynamical system (13) is asymptotically stable, implying that network (1) achieves synchronization.

Remark 6. For a dynamical network consisting of $N$ nodes, the number of possible coupling matrices which represent the coupling configurations of the network is finite. Therefore, one may define

$$
\lambda^{\max }=\max _{k=1,2, \ldots} \lambda_{2}\left(t_{k}\right), \quad \lambda^{\min }=\min _{k=1,2, \ldots} \lambda_{N}\left(t_{k}\right),
$$

and $\lambda=\max \left\{\left(1+c \lambda^{\max }\right)^{2},\left(1+c \lambda^{\min }\right)^{2}\right\}$. It is obvious that $\lambda\left(t_{k}\right) \leq \lambda$ for all $k=1,2, \ldots$.

Remark 7. The synchronizability of network (1) is determined by the second largest eigenvalue $\lambda_{2}\left(t_{k}\right)$ and the smallest eigenvalue $\lambda_{N}\left(t_{k}\right)$ of the coupling matrix, the coupling strength $c$, and impulsive intervals $\sigma_{k}=t_{k+1}-t_{k}, k=1,2, \ldots$.

\section{Numerical Simulations}

In this section, two illustrative examples about the chaotic Chua system and Lorenz system, respectively, are given to demonstrate the theoretical results obtained above. Without loss of generality, let the impulses be equidistant and separated by a constant interval $\sigma>0$.

Example 1. The chaotic Chua system is used as nodes of a dynamical network, which is described by

$$
\left(\begin{array}{c}
\dot{x} \\
\dot{y} \\
\dot{z}
\end{array}\right)=\left(\begin{array}{c}
\alpha[y-x-\varphi(x)] \\
x-y+z \\
-\beta y
\end{array}\right),
$$

where $\alpha, \beta$ are two parameters and $\varphi(x)=b x+0.5(a-$ $b)(|x+1|-|x-1|)$ represents a piecewise-linear diode, where
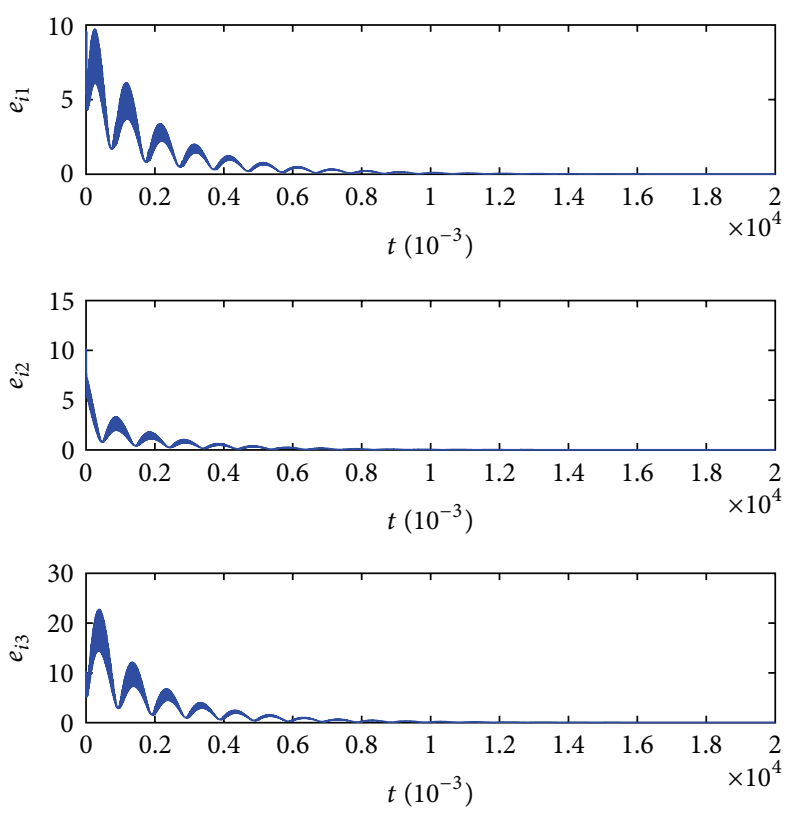

Figure 1: $N=100, \sigma=0.01$, and $c=0.01$.

$a<b<0$ are two constants. It is well known that Chua system is chaotic when $\alpha=9.22, \beta=15.99, a=-1.25$, and $b=-0.76$.

The intermittently coupled dynamical network is described by

$$
\begin{gathered}
\dot{x}_{i}(t)=f\left(x_{i}(t)\right), \quad t \neq t_{k}, \\
\Delta x_{i}\left(t_{k}\right)=c \sum_{j=1}^{N} a_{i j}\left(t_{k}\right) x_{j}\left(t_{k}\right), \quad t=t_{k}, k=1,2, \ldots,
\end{gathered}
$$

where

$$
f\left(x_{i}(t)\right)=\left(\begin{array}{c}
\alpha\left[x_{i 2}-x_{i 1}-\varphi\left(x_{i 1}\right)\right] \\
x_{i 1}-x_{i 2}+x_{i 3} \\
-\beta x_{i 2}
\end{array}\right) .
$$

The outer-coupling matrix $A\left(t_{k}\right)=\left(a_{i j}\left(t_{k}\right)\right) \in R^{N \times N}$ represents the coupling configuration of the dynamical network at instant $t_{k}$ and is defined as follows.

When $k$ is odd, the dynamical network has nearestneighbor coupling, so

$$
A\left(t_{k}\right)=A_{1}=\left(\begin{array}{cccccc}
-2 & 1 & 0 & \cdots & 0 & 1 \\
1 & -2 & 1 & \cdots & 0 & 0 \\
\vdots & \vdots & \vdots & \vdots & \vdots & \vdots \\
1 & 0 & 0 & \cdots & 1 & -2
\end{array}\right) .
$$

When $k$ is even, the dynamical network has star coupling, so

$$
A\left(t_{k}\right)=A_{2}=\left(\begin{array}{cccccc}
-N+1 & 1 & 1 & \cdots & 1 & 1 \\
1 & -1 & 0 & \cdots & 0 & 0 \\
\vdots & \vdots & \vdots & \vdots & \vdots & \vdots \\
1 & 0 & 0 & \cdots & 0 & -1
\end{array}\right) .
$$



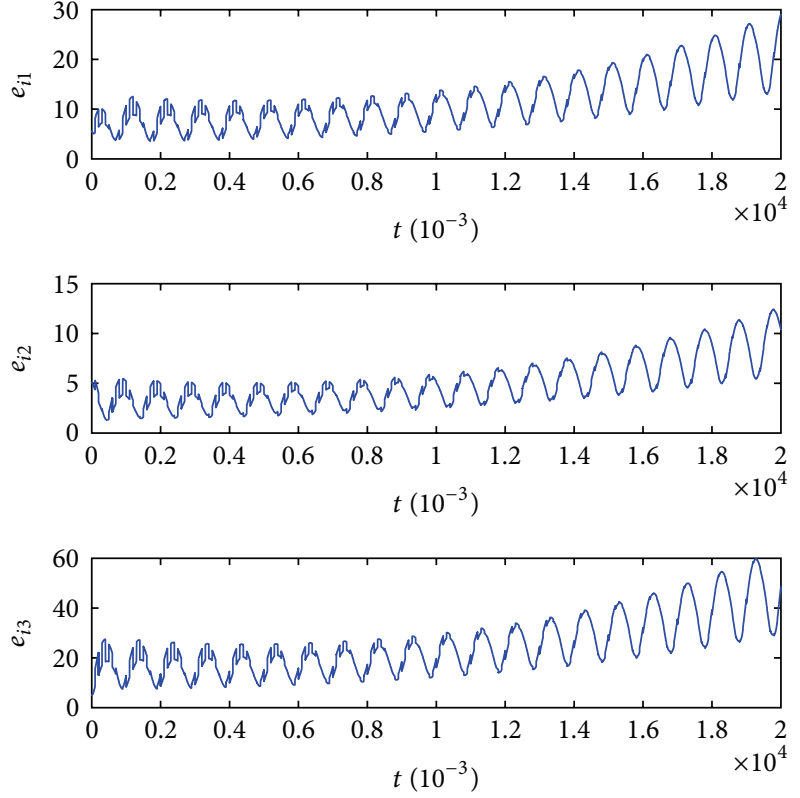

Figure 2: $N=100, \sigma=0.05$, and $c=0.01$.
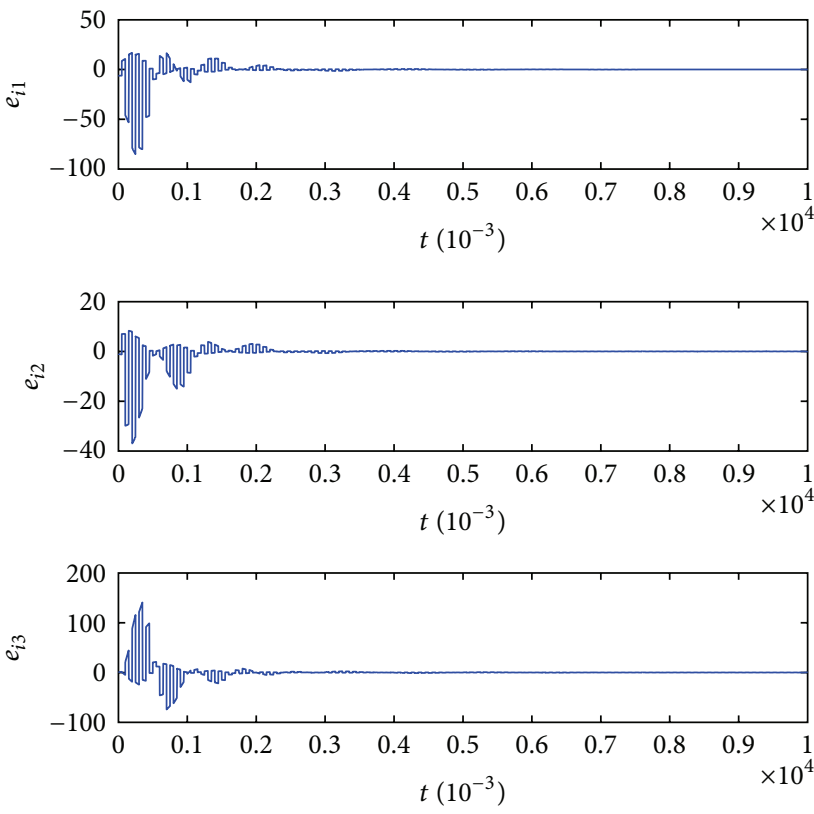

Figure 3: $N=100, \sigma=0.05$, and $c=0.05$.

In the simulation, we choose $N=100$. The initial values of these systems are chosen randomly from interval $[-20,20]$. For a small impulsive interval $\sigma>0$, the synchronization of network (29) can be achieved by choosing an appropriate coupling strength $c$. The numerical simulation results are shown in Figures 1-5, where $e_{i j}=\sum_{i=2}^{N}\left|x_{i j}-x_{1 j}\right| /(N-1), \quad j=$ $1,2,3$.

In Figure $1, \sigma=0.01$ and $c=0.01$. One can see that the state errors between node 1 and node $i, i=2,3, \ldots, 100$, tend to zero asymptotically as time evolves, implying that network (29) achieves synchronization. When $\sigma=0.05$ and
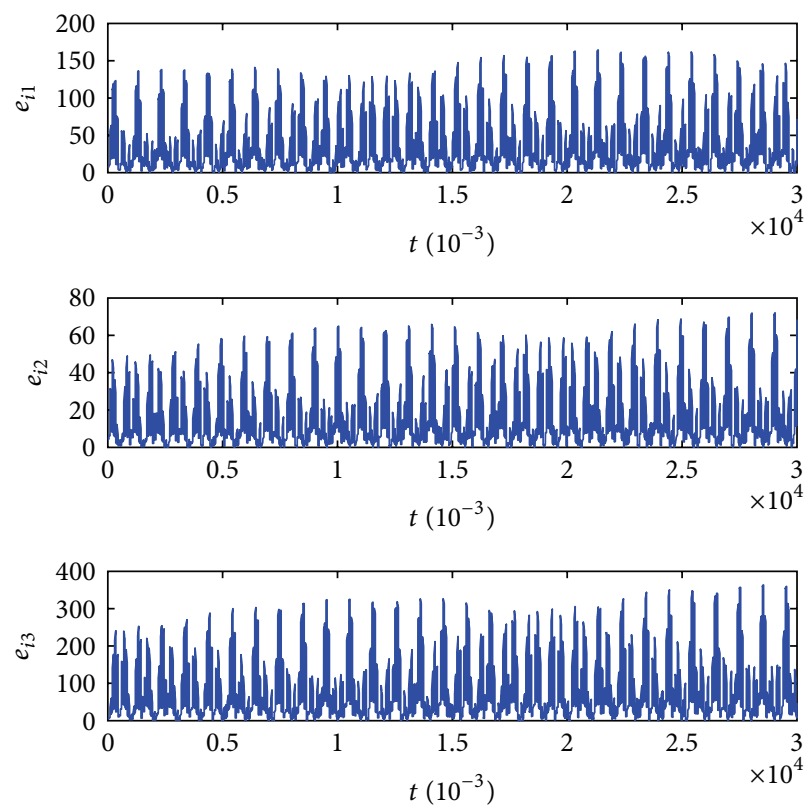

Figure 4: $N=100, \sigma=0.05$, and $c=0.06$.
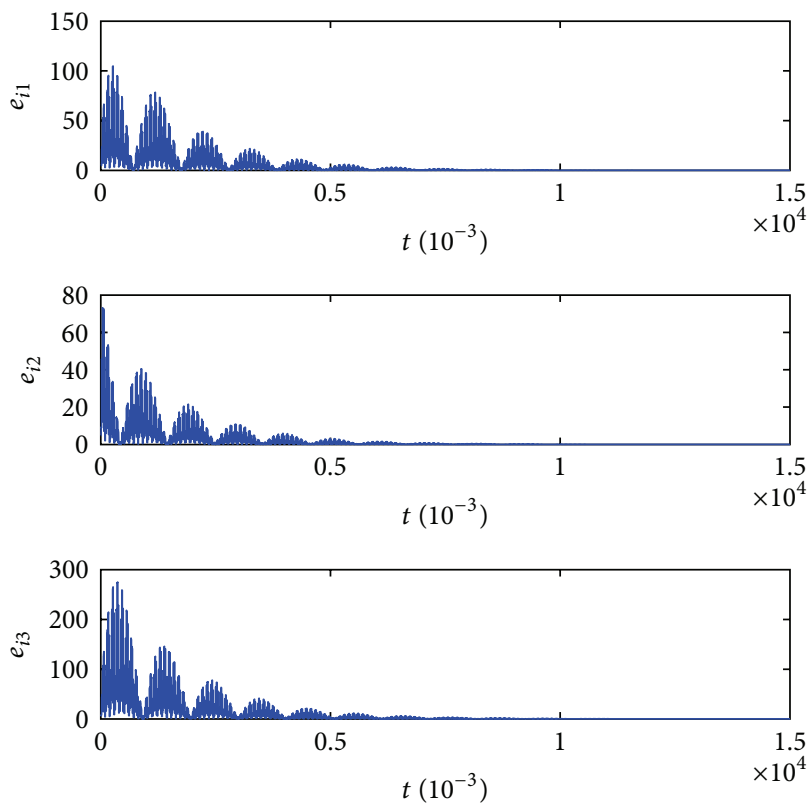

FIGURE 5: $N=100, \sigma=0.01$, and $c=0.06$.

$c=0.01$, network (29) cannot achieve synchronization, as shown in Figure 2. Figure 3 shows that network (29) achieves synchronization with $\sigma=0.05$ and $c=0.05$. Figure 4 shows that network (29) cannot achieve synchronization where $\sigma=$ 0.05 and $c=0.06$. Figure 5 shows that network (29) achieves synchronization with $\sigma=0.01$ and $c=0.06$. 

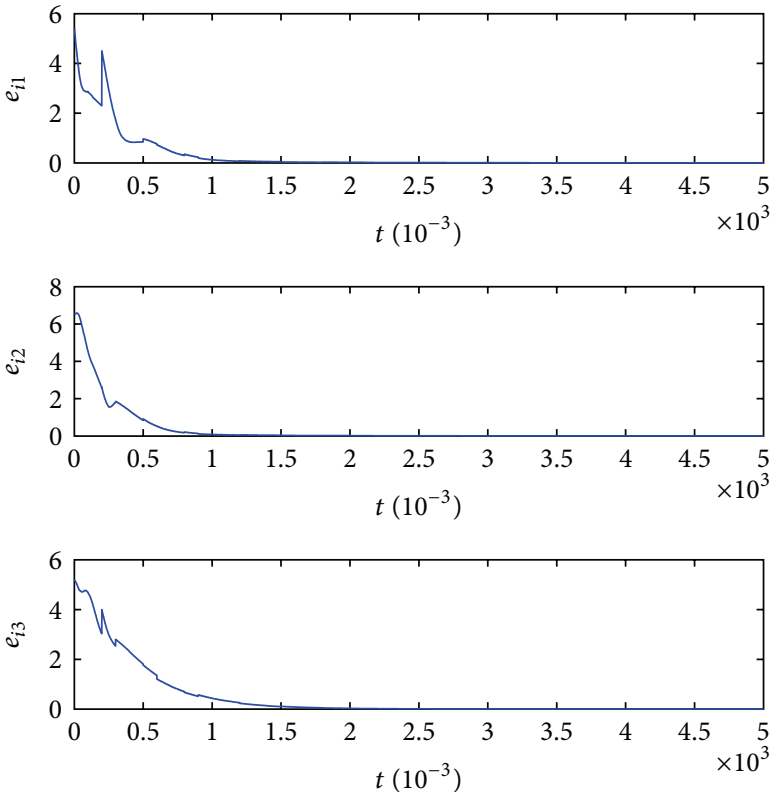

Figure 6: $N=200, \sigma=0.1$, and $c=0.01$.
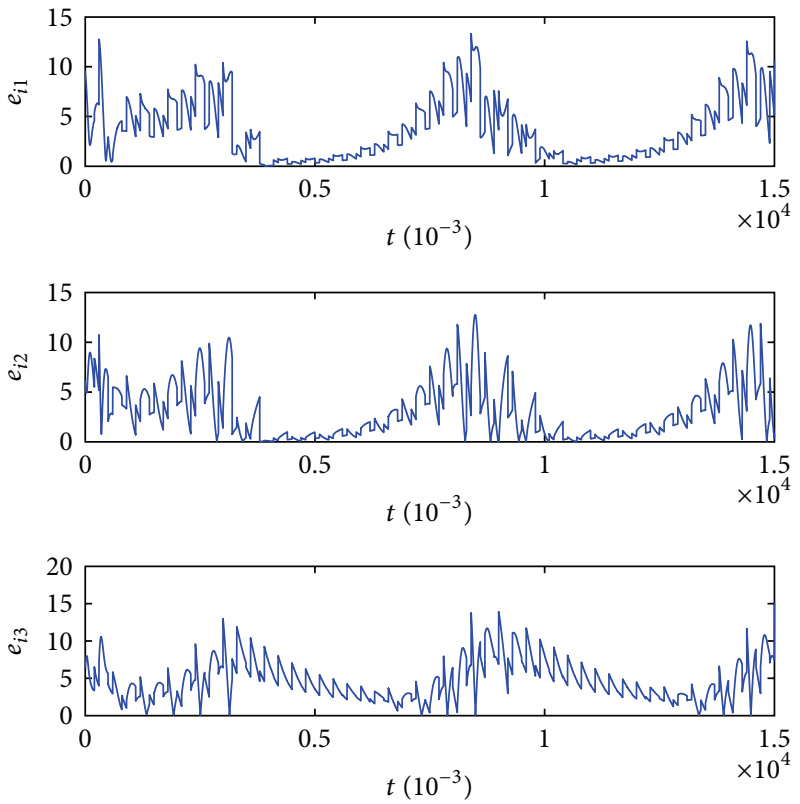

Figure 7: $N=200, \sigma=0.1$, and $c=0.015$.

Example 2. The chaotic Lorenz system is used as nodes of a dynamical network, which is described by

$$
\left(\begin{array}{c}
\dot{x} \\
\dot{y} \\
\dot{z}
\end{array}\right)=\left(\begin{array}{c}
a(y-x) \\
c x-x z-y \\
x y-b z
\end{array}\right)
$$

with a chaotic attractor when $a=10, b=8 / 3$, and $c=28$.
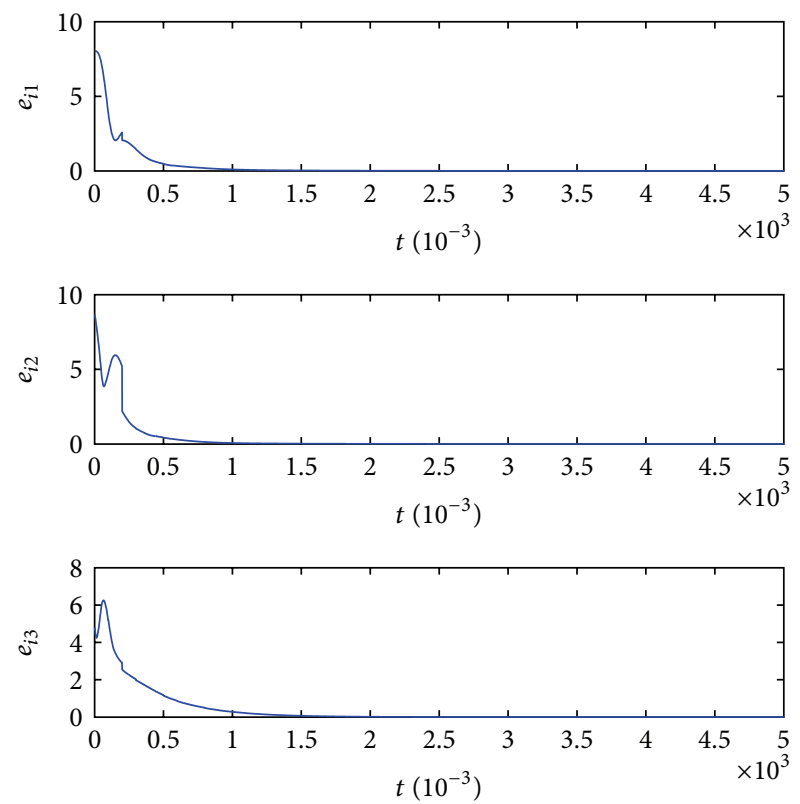

Figure 8: $N=50, \sigma=0.1$, and $c=0.015$.
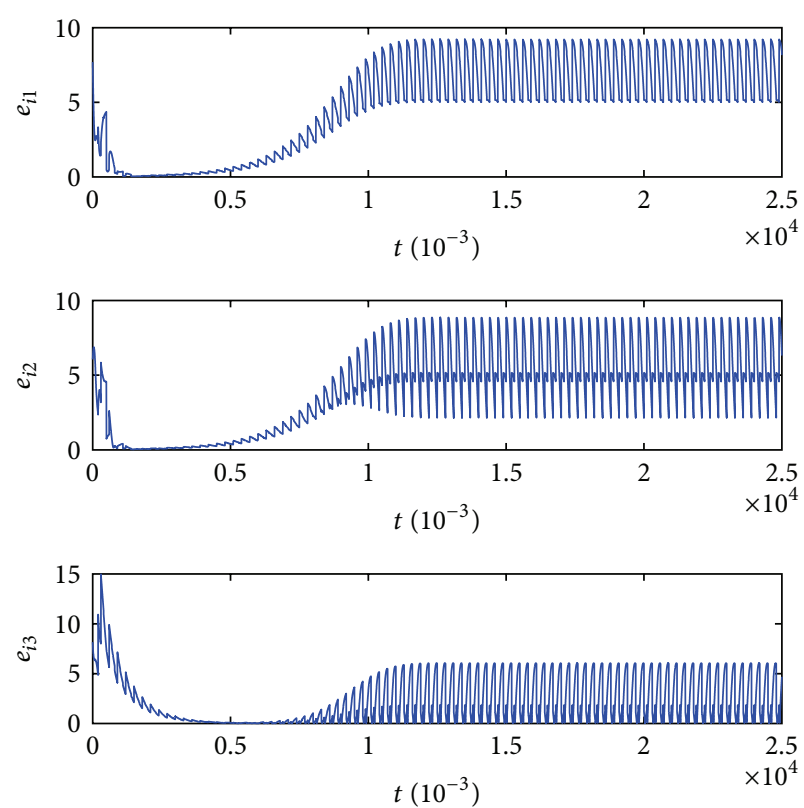

FIGURE 9: $N=50, \sigma=0.1$, and $c=0.055$.

The intermittently coupled dynamical network is described by

$$
\begin{aligned}
& \dot{x}_{i}(t)=f\left(x_{i}(t)\right), \quad t \neq t_{k}, k=1,2, \ldots, \\
& \Delta x_{i}\left(t_{k}\right)=c \sum_{j=1}^{N} a_{i j}\left(t_{k}\right) x_{j}\left(t_{k}\right), \quad t=t_{k},
\end{aligned}
$$



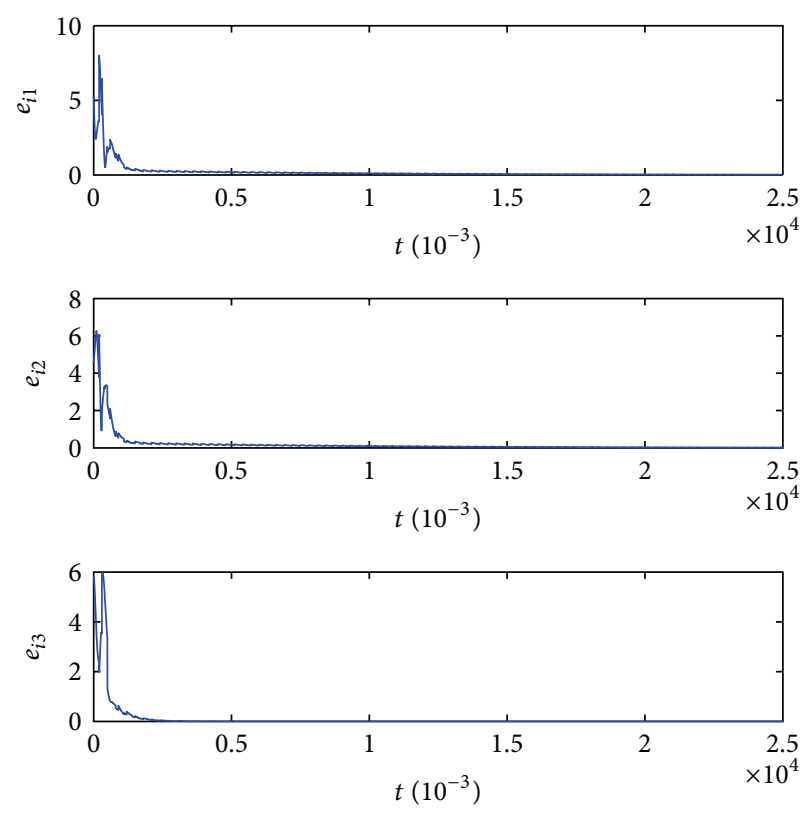

FIGURE 10: $N=50, \sigma=0.1$, and $c=0.048$.

where

$$
f\left(x_{i}(t)\right)=\left(\begin{array}{c}
a\left(x_{i 2}-x_{i 1}\right) \\
c x_{i 1}-x_{i 1} x_{i 3}-x_{i 2} \\
x_{i 1} x_{i 2}-b x_{i 3}
\end{array}\right) .
$$

Let $l \equiv k \bmod 3$. The outer-coupling matrix $A\left(t_{k}\right)=$ $\left(a_{i j}\left(t_{k}\right)\right) \in R^{N \times N}$ is defined as follows.

When $l=1$, the dynamical network has global coupling, and its outer-coupling matrix is

$$
A\left(t_{k}\right)=A_{g c}=\left(\begin{array}{ccccc}
-N+1 & 1 & 1 & \cdots & 1 \\
1 & -N+1 & 1 & \cdots & 1 \\
\vdots & \vdots & \vdots & \vdots & \vdots \\
1 & 1 & 1 & \cdots & -N+1
\end{array}\right) .
$$

When $l=2$, the dynamical network has nearest-neighbor coupling, with

$$
A\left(t_{k}\right)=A_{1}=\left(\begin{array}{cccccc}
-2 & 1 & 0 & \cdots & 0 & 1 \\
1 & -2 & 1 & \cdots & 0 & 0 \\
\vdots & \vdots & \vdots & \vdots & \vdots & \vdots \\
1 & 0 & 0 & \cdots & 1 & -2
\end{array}\right)
$$

When $l=0$, the dynamical network has star coupling, with

$$
A\left(t_{k}\right)=A_{2}=\left(\begin{array}{cccccc}
-N+1 & 1 & 1 & \cdots & 1 & 1 \\
1 & -1 & 0 & \cdots & 0 & 0 \\
\vdots & \vdots & \vdots & \vdots & \vdots & \vdots \\
1 & 0 & 0 & \cdots & 0 & -1
\end{array}\right) .
$$

In the simulation, we choose $\sigma=0.1$. The initial values of these systems are chosen randomly from interval $[-20,20]$. The numerical simulation results indicate that the synchronizability of network (34) is related to the coupling strength $c$ and the number of nodes $N$, as shown in Figures $6-10$, where $e_{i j}=\sum_{i=2}^{N}\left|x_{i j}-x_{1 j}\right| /(N-1), j=1,2,3$. For the coupling matrices of this network (34), the spectral width of eigenvalues will be broadened with increasing $N$. Therefore, the synchronizability of the network will be weakened if the node number increases sharply.

In Figure $6, N=200$ and $c=0.01$. One can see that the state errors tend to zero asymptotically as time evolves, implying that network (34) achieves synchronization. When $N=200$ and $c=0.015$, network (34) cannot achieve synchronization, as illustrated in Figure 7. When $N=50$ and $c=0.015$, network (34) achieves synchronization, as shown in Figure 8. When $N=50$ and $c=0.055$, network (34) cannot achieve synchronization, as shown in Figure 9. Figure 10 demonstrates that network (34) achieves synchronization when $N=50$ and $c=0.048$.

\section{Conclusions}

This paper investigates the synchronization phenomenon of an intermittently coupled dynamical network, in which the coupling among nodes can occur only at discrete instants and the coupling configuration of the network is varying at different instants. For such an intermittently coupled dynamical network consisting of identical nodes, based on the stability theory for impulsive differential equations, some synchronization conditions are derived. It is shown that the synchronizability of the network is related to the second largest eigenvalue and the smallest eigenvalue of the coupling matrix, the coupling strength, and the impulsive intervals. It is worth noting that the analytical results about the second largest and the smallest eigenvalues of the coupling matrix are consistent with most existing criteria for complex dynamical networks. Finally, by employing the chaotic Chua system and Lorenz system as nodes of a dynamical network, respectively, numerical simulations are carried out to illustrate and verify the theoretical results.

\section{Acknowledgments}

The authors gratefully acknowledge the support of the National Natural Science Foundation of China (NNSF) through Grant no. 10972011, the Natural Science Foundation of Hebei Province of China through Grant no. A2010001943, and the Hong Kong Research Grants Council through GRF Grant City U1114/11E.

\section{References}

[1] P. Erdős and A. Rényi, "On the evolution of random graphs," Publications of the Mathematical Institute of the Hungarian Academy of Sciences, vol. 5, pp. 17-61, 1960.

[2] D. J. Watts and S. H. Strogatz, "Collective dynamics of smallworld networks," Nature, vol. 393, no. 6684, pp. 440-442, 1998.

[3] M. E. J. Newman and D. J. Watts, "Scaling and percolation in the small-world network model," Physical Review E, vol. 60, no. 6, pp. 7332-7342, 1999. 
[4] M. E. J. Newman, "The structure and function of complex networks," SIAM Review, vol. 45, no. 2, pp. 167-256, 2003.

[5] A. L. Barabási and R. Albert, "Emergence of scaling in random networks," Science, vol. 286, no. 5439, pp. 509-512, 1999.

[6] A. L. Barabsi, R. Albert, H. Jeong, and G. Bianconi, "Power-law distribution of the world wide web," Science, vol. 287, no. 5461, p. 2115, 2000.

[7] R. Albert and A. L. Barabási, "Statistical mechanics of complex networks," Reviews of Modern Physics, vol. 74, no. 1, pp. 47-97, 2002.

[8] X. F. Wang and G. Chen, "Synchronization in scale-free dynamical networks: robustness and fragility," IEEE Transactions on Circuits and Systems I, vol. 49, no. 1, pp. 54-62, 2002.

[9] X. F. Wang and G. Chen, "Synchronization in small-world dynamical networks," International Journal of Bifurcation and Chaos in Applied Sciences and Engineering, vol. 12, no. 1, pp. 187192, 2002.

[10] T. Nishikawa, A. E. Motter, Y. Lai, and F. C. Hoppensteadt, "Heterogeneity in oscillator networks: are smaller worlds easier to synchronize?" Physical Review Letters, vol. 91, no. 1, Article ID 014101, pp. 1-4, 2003.

[11] V. N. Belykh, I. V. Belykh, and M. Hasler, "Connection graph stability method for synchronized coupled chaotic systems," Physica D, vol. 195, no. 1-2, pp. 159-187, 2004.

[12] I. Belykh, V. Belykh, and M. Hasler, "Generalized connection graph method for synchronization in asymmetrical networks," Physica D, vol. 224, no. 1-2, pp. 42-51, 2006.

[13] J. Lü, X. Yu, and G. Chen, "Chaos synchronization of general complex dynamical networks," Physica A, vol. 334, no. 1-2, pp. 281-302, 2004.

[14] C. Li and G. Chen, "Synchronization in general complex dynamical networks with coupling delays," Physica A, vol. 343, no. 1-4, pp. 263-278, 2004.

[15] J. Cao and J. Lu, "Adaptive synchronization of neural networks with or without time-varying delay," Chaos, vol. 16, no. 1, Article ID 013133, p. 6, 2006.

[16] W. Xia and J. Cao, "Adaptive synchronization of a switching system and its applications to secure communications," Chaos, vol. 18, no. 2, Article ID 023128, p. 15, 2008.

[17] W. Yu, G. Chen, J. Cao, J. Lu, and U. Parlitz, "Parameter identification of dynamical systems from time series," Physical Review E, vol. 75, no. 6, Article ID 067201, 2007.

[18] Z. Ma, Z. Liu, and G. Zhang, "A new method to realize cluster synchronization in connected chaotic networks," Chaos, vol. 16, no. 2, Article ID 023103, p. 9, 2006.

[19] J. G. Restrepo, E. Ott, and B. R. Hunt, "Emergence of synchronization in complex networks of interacting dynamical systems," Physica D, vol. 224, no. 1-2, pp. 114-122, 2006.

[20] J. Zhou and T. Chen, "Synchronization in general complex delayed dynamical networks," IEEE Transactions on Circuits and Systems I, vol. 53, no. 3, pp. 733-744, 2006.

[21] J. Zhou, T. Chen, and L. Xiang, "Adaptive synchronization of coupled chaotic delayed systems based on parameter identification and its applications," International Journal of Bifurcation and Chaos in Applied Sciences and Engineering, vol. 16, no. 10, pp. 2923-2933, 2006.

[22] J. Zhou, T. Chen, and L. Xiang, "Robust synchronization of delayed neural networks based on adaptive control and parameters identification," Chaos, Solitons and Fractals, vol. 27, no. 4, pp. 905-913, 2006.
[23] Z. Guan, J. Hill, and X. Shen, "On hybrid impulsive and switching systems and application to nonlinear control," IEEE Transactions on Automatic Control, vol. 50, no. 7, pp. 1058-1062, 2005.

[24] Z. Guan and H. Zhang, "Stabilization of complex network with hybrid impulsive and switching control," Chaos, Solitons \& Fractals, vol. 37, no. 5, pp. 1372-1382, 2008.

[25] B. Liu, X. Liu, G. Chen, and H. Wang, "Robust impulsive synchronization of uncertain dynamical networks," IEEE Transactions on Circuits and Systems I, vol. 52, no. 7, pp. 1431-1441, 2005.

[26] G. Zhang, Z. Liu, and Z. Ma, "Synchronization of complex dynamical networks via impulsive control," Chaos, vol. 17, no. 4, Article ID 043126, p. 9, 2007.

[27] J. Lu, D. W. C. Ho, and J. Cao, "A unified synchronization criterion for impulsive dynamical networks," Automatica, vol. 46, no. 7, pp. 1215-1221, 2010.

[28] Y. Yang and J. Cao, "Exponential synchronization of the complex dynamical networks with a coupling delay and impulsive effects," Nonlinear Analysis. Real World Applications. An International Multidisciplinary Journal, vol. 11, no. 3, pp. 1650-1659, 2010.

[29] Y. Tang, S. Y. S. Leung, W. K. Wong, and J. Fang, "Impulsive pinning synchronization of stochastic discrete-time networks," Neurocomputing, vol. 73, no. 10-12, pp. 2132-2139, 2010.

[30] J. Zhou, Q. Wu, and L. Xiang, "Pinning complex delayed dynamical networks by a single impulsive controller," IEEE Transactions on Circuits and Systems I, vol. 58, no. 12, pp. 28822893, 2011.

[31] X. Han, J.-A. Lu, and X. Wu, "Synchronization of impulsively coupled systems," International Journal of Bifurcation and Chaos in Applied Sciences and Engineering, vol. 18, no. 5, pp. 1539-1549, 2008.

[32] W. Sun, F. Austin, J. Lü, and S. Chen, "Synchronization of impulsively coupled complex systems with delay," Chaos, vol. 21, no. 3, Article ID 033123, 2011.

[33] T. Yang, Impulsive Control Theory, Springer, Berlin, Germany, 2001.

[34] V. Lakshmikantham, D. D. Bainov, and P. S. Simeonov, Theory of Impulsive Differential Equations, World Scientific, Singapore, 1989. 


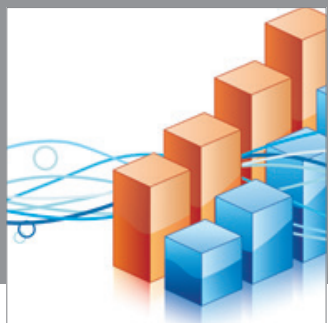

Advances in

Operations Research

mansans

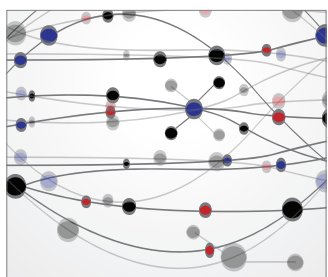

The Scientific World Journal
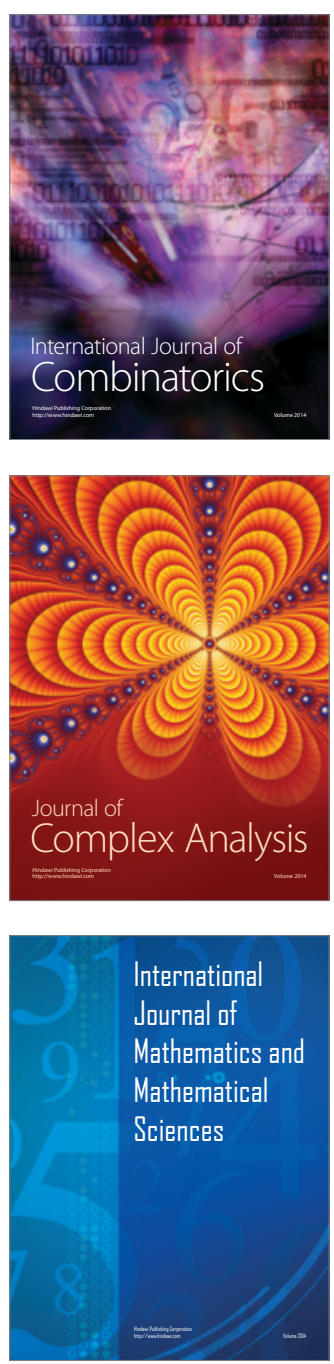
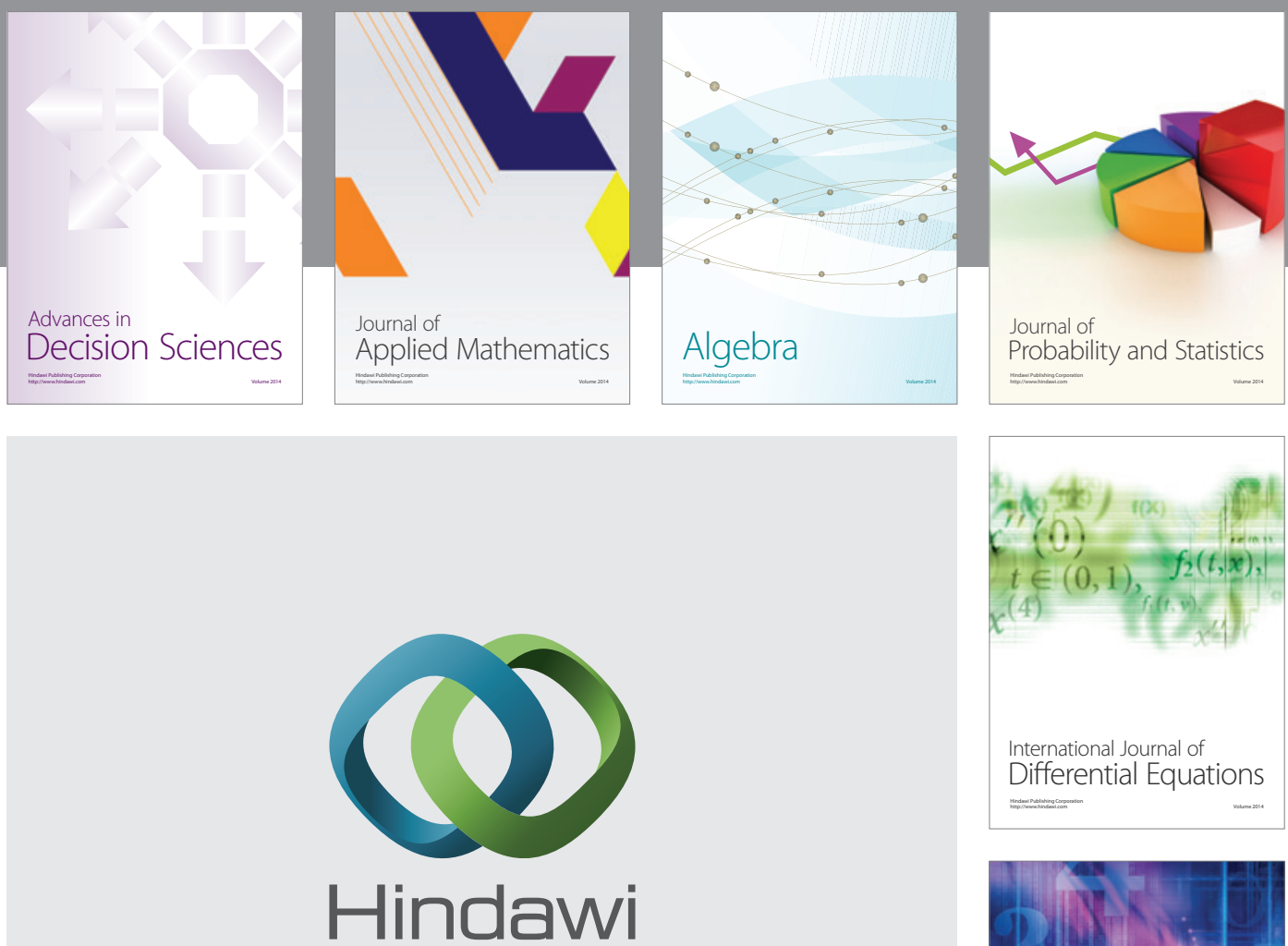

Submit your manuscripts at http://www.hindawi.com
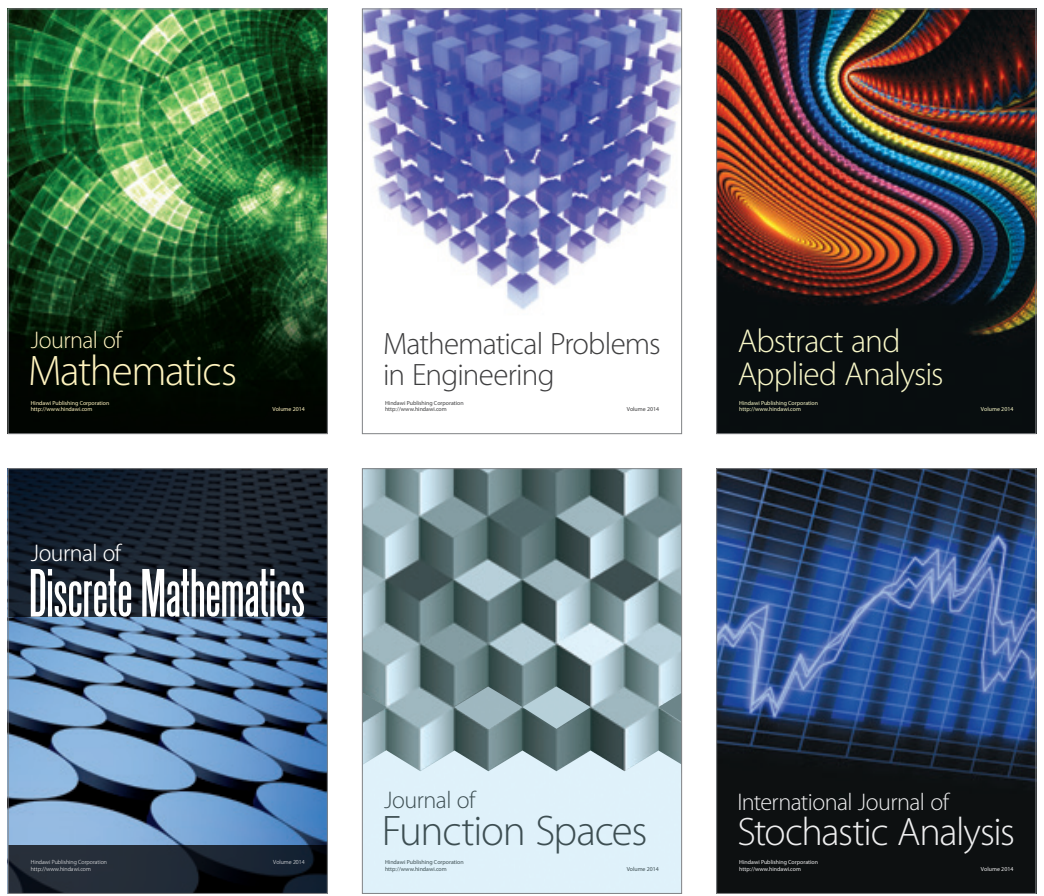

Journal of

Function Spaces

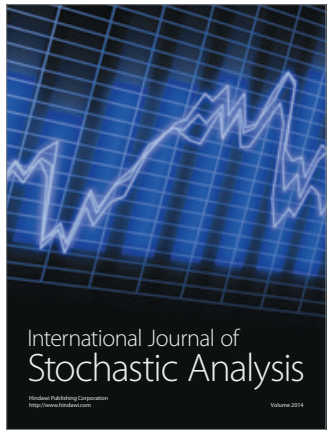

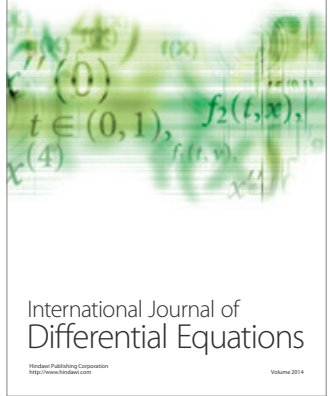
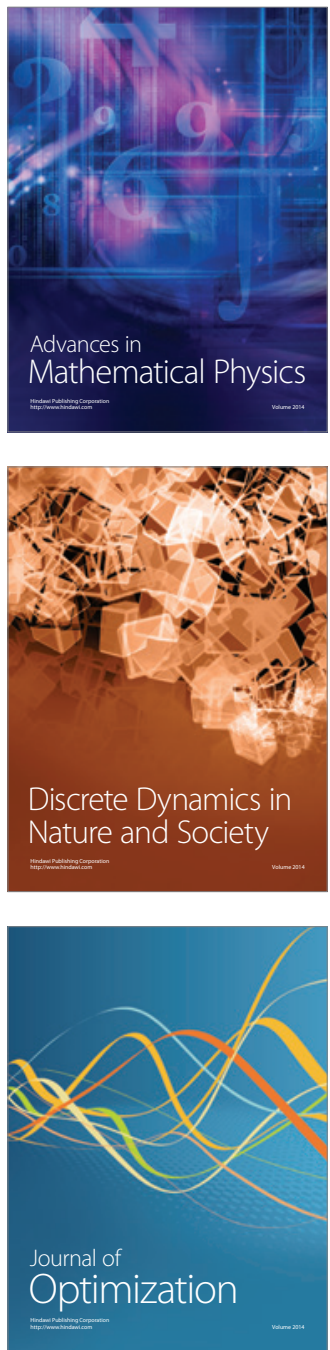